\title{
La 'organización que aprende' y su aporte al proceso de cambio
}

Arturo Solf Zárate

El presente trabajo se inicia con precisiones sobre las semejanzas y diferencias del aprendizaje individual frente al de grupos y de todo el sistema organizacional desde una perspectiva conductual, como una introducción al análisis sobre la 'organización que aprende' (learning organization), la cual se refiere a un tipo ideal de organización que otorga prioridad al aprendizaje de todos sus miembros $y$ del sistema total.

Además del diagnóstico y desarrollo de una 'organización que aprende', proporciona información sobre el instrumento Dimensions of the Learning Organization Questionnaire (DLOQ) elaborado por Watkins y Marsick (1998) con la finalidad de medir la cultura de aprendizaje, que podría ser aplicado con cualquier otro enfoque de cambio organizacional.

Aprendizaje Organizacional / Organización que Aprende / ISO 9001 / TQM / Indagación Apreciativa (IA) / Cambio Organizacional

\section{The learning organization and his contributions for the organizational change}

In this article the author begins by discussing the similarity and differences between individual learning, and organizational system learning from a behavioral standpoint as an introduction to the analysis of Learning Organization, an ideal type of organization that focuses on the learning of each of its members and of the whole system. The article then presents a diagnosis and elaboration of a Learning Organization using the Dimensions of the Learning Organization Questionnaire (DLOQ), an instrument developed by Watkins and Marsick (1998) aimed at measuring the Learning Culture. This instrument can be applied in any other approach of organizational change.

Organizational Learning / Learning Organization / ISO 9001 / TQM / Appreciative Inquiry (Al) / Organizational Change 


\section{IMPORTANCIA DEL APRENDIZAJE EN LAS ORGANIZACIONES}

Actualmente, las organizaciones laborales se enfrentan a la necesidad de una adaptación continua para estar acordes con las exigencias de un contexto más competitivo; para ello, necesitan realizar modificaciones en sus estructuras organizacionales, tecnología, forma de administrar al personal, etcétera (Yeo, 2005). Dicha adaptación implica aprendizaje, entendiéndose este como “... un cambio relativamente permanente en la conducta y en las capacidades humanas producto de la experiencia y la práctica" (Landy, 2005, p. 293). Las organizaciones tienen que aprender a responder adecuadamente a las exigencias de su medio, y deben hacerlo más rápido que sus competidores (Guns, 1996).

Si bien se puede ubicar el inicio del estudio de las organizaciones desde la perspectiva del aprendizaje con el aporte de March y Simon (1958) que continuó con el de Argyris y Schön (1978), es a partir de la publicación de La quinta disciplina de Peter Senge (1992) que el interés sobre el tema se incrementó exponencialmente en los practicantes y teóricos vinculados a las organizaciones laborales. Una expresión de este interés lo podemos verificar en el buscador Google, donde encontramos -hasta la fecha de redacción de este trabajo- más de novecientas mil entradas registradas para Learning Organization.
Otro factor que ha permitido dar más importancia al aprendizaje en las organizaciones ha sido la necesidad de superar el entrenamiento como responsabilidad exclusiva del centro de capacitación, por un enfoque de aprendiza$j e$ en todas las actividades que realiza el empleado, y especialmente en la ejecución de sus tareas (Poell, Van Dam, \& Van den Berg, 2004; Sonnetang, Niessen, \& Ohly, 2004).

\section{APRENDIZAJE ORGANIZACIONAL Y ORGANIZACIONES QUE APRENDEN}

Es necesario diferenciar entre 'aprendizaje organizacional' (organizational learning) y 'organizaciones que aprenden' (learning organization). El primer caso se refiere a las modificaciones que efectúa la organización en su forma de realizar sus actividades a partir de sus experiencias, mientras que la organización que aprende es un tipo ideal de organización en la que el aprendizaje ocupa un rol importante (Swieringa \& Wierdsma, 1995). Argyris (2001), al analizar la bibliografía que presta atención al aprendizaje en las organizaciones, distingue dos vertientes: a) sobre la 'organización que aprende', que es prescriptiva, más orientada a la práctica, y b) sobre el 'aprendizaje organizacional', más descriptiva, producida por los académicos que indagan sobre cómo aprenden las personas y grupos en contextos organizacionales.

Algunos autores consideran ambas denominaciones como sinónimos 
(Watkins \& Marsick, 1998; Chen, 2005), pero es necesario diferenciarlas, por la sencilla razón de que existe 'aprendizaje organizacional' en la medida en que cualquier grupo o todo el sistema introducen cambios relativamente estables en la forma de efectuar sus actividades, mientras que las 'organizaciones que aprenden' se caracterizan por fomentar, como ventaja estratégica, el aprendizaje de sus miembros y el sistema total. (Solf, 2003). De este modo, la expresión 'desarrollo de una organización que aprende' implica un enfoque de cambio sistemático e intencional orientado a conseguir que una organización “... aprenda y continuamente expanda su capacidad para crear el futuro" (Senge, 1992, p. 24).

El aprendizaje organizacional y las organizaciones que aprenden, si bien se refieren a dos entidades diferentes, están interrelacionadas, de manera que una organización que aprende canaliza de manera intencional el aprendizaje organizacional. Puede existir aprendizaje organizacional sin una organización que aprende, pero no lo contrario.

Los especialistas en el tema han proporcionado diferentes definiciones sobre la organización que aprende, algunas de las cuales, las más representativas y que otorgan prioridad a la conducta, presentamos a continuación:

Senge, en su libro mencionado, afirma que las organizaciones que aprenden son aquellas “... donde la gente expande continuamente su aptitud para crear los resultados que desea, donde se cultivan nuevos y expansivos patrones de pensamiento, donde la aspiración colectiva queda en libertad, y donde la gente continuamente aprende a aprender en conjunto" (p. 11).

Braham (1995) proporciona una definición más corta: "una organización que aprende es aquella que prioriza el aprendizaje" (p. 49).

Para Pedler, Burgoyne y Boydell (1997) una organización que aprende es aquella que "... facilita el aprendizaje de todos sus miembros, y continuamente se transforma a sí misma" (p. 3).

Otros especialistas han definido la organización que aprende desde el punto de vista del conocimiento (Fruint, 2000; Nonaka, 1999; Krogh, Ichijo, \& Nonaka, 2001; Yeung et al., 2000; Choo, 1999). Así, para Yeung et al. (2000) dichas organizaciones se caracterizan por tener la capacidad fundamental “... para generar y generalizar ideas con impacto (cambio) a través de varios límites de la organización (aprendizaje) por medio de iniciativas y prácticas específicas de la administración (capacidad)" (p. 11). Chen (2005), quien usa como sinónimos 'aprendizaje organizacional' y 'organización que aprende', afirma que:

El aprendizaje organizacional se refiere a los procesos a través del cual una organización continuamente adquiere nuevos ajustes y conocimientos a fin de adaptarse exitosamente a los cambios medioambientales internos y externos, y para mantener un desarrollo y existencia sostenible (p. 4). 
Al considerar el conocimiento nos enfrentamos al problema de su definición, lo cual es complicado y constituye uno de los objetivos principales de la epistemología. En este trabajo asumimos la propuesta de Koulopoulos y Frappaolo (2000), para quienes el conocimiento es la información útil para efectuar una acción, de manera que no tendría mayor relevancia el simple conocer sin hacer. Otra dimensión de análisis es la relación entre conocimiento y aprendizaje. El conocimiento puede ser tanto consecuencia como causa del aprendizaje, de modo que un resultado final de la reflexión sobre una conducta exteriorizada podría ser el conocimiento, y a la vez un conocimiento es capaz de propiciar una nueva experiencia (Kolb, Rubin, \& McIntyre, 1974; Bhatt, 2000).

Para tener una mejor comprensión del aprendizaje organizacional, se indagará a continuación acerca de las diferencias y semejanzas con el aprendizaje individual.

\section{APRENDIZAJE INDIVIDUAL Y ORGANIZACIONAL}

La definición general de aprendizaje como el cambio de conducta a partir de la experiencia puede ser útil para describir el aprendizaje en los ámbitos individual, grupal y organizacional. Los tres ámbitos son iguales desde esta perspectiva, pero cuando se indaga sobre los procesos que intervienen en cada uno de ellos ya no es útil dicha definición. Así, el aprendizaje individual se produce a través del cerebro de los sujetos, lo que no sucede en el aprendizaje grupal $\mathrm{u}$ organizacional. Además, el aprendizaje de muchos sujetos no implica necesariamente un mejor desempeño de un grupo u organización. Esto nos indica que existen diferencias entre el aprendizaje individual y el organizacional, lo cual ha fomentado posiciones contradictorias en su análisis (Argyris, 2001).

Yeung et al. (2000) establecen un distingo entre aprendizaje individual y aprendizaje organizacional de la siguiente manera:

El aprendizaje personal ocurre cuando los miembros de la organización adquieren conocimientos por medio de la educación, la experiencia o la experimentación. Las organizaciones aprenden cuando sus sistemas y su cultura conservan estos conocimientos y los transfieren a partir de los individuos (p. 9).

Desde una postura conductual, Swieringa y Wierdsma (1995) afirman que existe aprendizaje organizacional solo cuando "... un cambio en el comportamiento de un individuo tiene efecto en el de otros" (p. 37). De este modo, el aprendizaje organizacional implica influencia de un sujeto en el accionar de otro u otros, lo cual puede suceder directa o indirectamente, a través de la cultura (valores predominantes) o de los reglamentos, disposiciones y normas de trabajo. 
Algunos autores, como Dixon (1999) o Alcover y Gil (2002), identifican el aprendizaje grupal y organizacional como la creación colectiva de conocimiento, pero consideramos que es una definición incompleta al no precisar que el nuevo conocimiento origina cambios relativamente estables.

Para resumir, podemos decir que existe aprendizaje organizacional cuando los aportes de los sujetos permiten un cambio que puede mantenerse en el futuro $\sin$ la presencia de aquellos; cuando el aporte de los individuos está asimilado en la organización, de manera que “... las lecciones aprendidas son incorporadas por largo plazo en la cultura y modus operandi de la organización" (Ben-Horin, Lipshitz, \& Popper, 2004, p. 456). Otro aspecto que se debe resaltar es la naturaleza siempre social del aprendizaje organizacional, al requerir por lo menos dos personas, lo cual no sucede en el aprendizaje individual.

Un tema vinculado con la relación entre el aprendizaje individual y el aprendizaje organizacional es la crítica de antropomorfismo al atribuir a la organización una característica propia de las personas: el aprender. Se afirma que aprenden las personas, no las organizaciones (Argyris, 2001). Friedman et al. (2005) recomiendan que para superar esta crítica se consideren mecanismos organizacionales tangibles, como la retroinformación. Otros han intentado superar el problema al preci- sar que el concepto aprendizaje organizacional es solo una metáfora (Gore y Dunlap, 2006).

\section{CALIDAD DEL APRENDIZAJE ORGANIZACIONAL}

Además de las diferencias entre el aprendizaje individual y el organizacional, según Ben-Horin et al. (2004) este último también se puede diferenciar de acuerdo con su calidad. Estos autores establecen un distingo entre aprendizaje organizacional de alta y baja calidad. Sobre la base de una investigación acerca de experiencias de aprendizaje exitosas y no exitosas en la fuerza aérea israelí, se indica que el aprendizaje organizacional de alta calidad es aquel que básicamente permite el logro de objetivos deseados y útiles para la organización, y las conductas establecidas tienen estabilidad en el tiempo; mientras que el aprendizaje organizacional de baja calidad no posee estas características; se puede aprender algo útil pero su aplicación no tiene continuidad, o se aprenden conductas disfuncionales para la organización, que perduran a lo largo del tiempo.

Ben-Horin et al. (2004), sobre la base de su investigación concluyeron que un aspecto importante para el aprendizaje organizacional de alta calidad fue la participación y el compromiso para el aprendizaje de parte de los involucrados. Esto se logró gracias a la cultura de aprendizaje y a los líderes 
que apoyaron el aprendizaje y la seguridad psicológica del personal. La cultura de aprendizaje promueve los siguientes valores: proporcionar información válida y verificable, realizar un aprendizaje continuo, asumir la responsabilidad de las acciones, estar dispuesto a aceptar el escrutinio de los demás sobre el desempeño individual y estar orientado a los objetivos sin interesar los niveles o las personas involucradas.

\section{DIAGNÓSTICO DE UNA ORGANIZACIÓN QUE APRENDE}

No es posible medir directamente el constructo 'organización que aprende' sino a través de ciertos indicadores. Es algo parecido a lo que sucede con la medición de la inteligencia, la motivación, etcétera, para lo cual se recurre a mediciones indirectas, como los cuestionarios o pruebas psicológicas.

En el ámbito de la organización que aprende se han elaborado muchas teorías pero pocos instrumentos para su medición (Thomas \& Allen, 2006). Moilanen (2005) identifica ocho instrumentos, y al analizarlos destaca el de Tannembaum (1997) y el de Watkins y Marsick (1998), al que considera “... probablemente el cuestionario más completo..." (p. 73). Al cuestionario de Mayo y Lank (1994) lo considera muy extenso (187 ítems). A la lista de Moilanen habría que incluir el elaborado por O'Brien (1994), quien basándose en un análisis de la biblio- grafía identificó doce dimensiones y elaboró su cuestionario con un total de 60 ítems.

El cuestionario elaborado por Watkins y Marsick (1998) es Dimensions of the Learning Organization Questionnaire (DLOQ), que además de ser el más completo según Moilanen (2005), también ha sido objeto de mayor investigación y validación. Por dichas razones presentamos a continuación más detalles sobre este.

El DLOQ se elaboró para medir la cultura de aprendizaje de una organización que aprende. Está conformada por siete dimensiones, además de dos escalas para medir el desempeño financiero y el desempeño del conocimiento (Marsick \& Watkins, 2003). Las siete dimensiones y sus definiciones son las siguientes:

- Crear oportunidades de aprendizaje continuo. El trabajo está diseñado de modo que la gente pueda aprender en el trabajo; se otorgan oportunidades para un continuo aprendizaje y crecimiento.

- Promover la indagación y el diálogo. La gente adquiere habilidades de razonamiento productivo para expresar sus puntos de vista, y escuchar e indagar los pensamientos de los demás; la cultura apoya el cuestionamiento, el feedback y la experimentación.

- Animar la colaboración y aprendizaje de equipo. El trabajo es diseña- 
do para usar grupos y así acceder a diferentes modos de pensamiento; se espera que en los grupos se aprenda y trabaje conjuntamente.

- Crear sistemas para capturar y compartir aprendizaje. Se crean sistemas de alta y baja tecnología para compartir el aprendizaje e integrarlos al trabajo; se otorga acceso a los sistemas; los sistemas se mantienen.

- Empoderar a la gente hacia una visión colectiva. La gente es involucrada con el fin de que establezca, se apropie e implemente una visión conjunta; la responsabilidad es distribuida lo más cerca posible de los niveles de decisión, de modo que la gente esté motivada para aprender lo que tiene que hacer.

- Conectar la organización con su medio ambiente. Se ayuda a las personas a percibir los efectos de su trabajo en la empresa; la gente vigila su medio ambiente y usa la infor- mación para ajustar sus prácticas laborales; la organización está vinculada con sus comunidades.

- Proporcionar liderazgo estratégico para el aprendizaje. Los líderes modelan y apoyan el aprendizaje; el liderazgo usa estratégicamente el aprendizaje para obtener mejores resultados.

Las siete dimensiones incluyen un total de 43 ítems, que abarca los niveles individual, grupal y organizacional (Marsick \& Watkins, 2003). Yang (2003) demostró que el DLOQ tiene buena validez de predicción, y los coeficientes alfa para la confiabilidad de las dimensiones tenían un rango de .80 a .87. Además, este autor obtuvo una versión abreviada de 21 ítems, y de estos identificó siete para obtener un solo puntaje de cultura de aprendizaje. En la tabla 1 se incluyen los ítems de la forma abreviada y los siete para un puntaje total (identificados con asterisco).

Tabla 1

Ítems de la forma abreviada del DLOQ

\footnotetext{
Aprendizaje continuo

- En mi organización, la gente se ayuda mutuamente a aprender.

- En mi organización, se le da tiempo a la gente para apoyar su aprendizaje.

- $\quad\left(^{*}\right)$ En mi organización, se recompensa a la gente por aprender.

Indagación y diálogo

- En mi organización, se da retroalimentación mutua de manera abierta y honesta.

- En mi organización, cada vez que alguien expresa sus opiniones también pregunta qué piensan los demás al respecto.

- $\quad\left(^{*}\right)$ En mi organización, la gente se esfuerza por establecer vínculos de confianza entre ellos.
} 
Continuación

\section{Colaboración y trabajo de equipo}

- En mi organización, los grupos/equipos tienen la libertad de modificar sus metas cuando sea necesario.

- $\quad\left(^{*}\right)$ En mi organización, los grupos/equipos reexaminan sus pensamientos como resultado de discusiones de grupo o información recogida.

- En mi organización, los grupos/equipos tienen confianza en que la organización actuará de acuerdo con sus recomendaciones.

\section{Sistema para capturar aprendizaje}

- Mi organización crea sistemas para medir la diferencia entre el rendimiento actual y el previsto.

- $\quad{ }^{*}$ ) Mi organización pone su conocimiento y sabiduría a disposición de todos los empleados.

- Mi organización mide los resultados del tiempo y recursos gastados en el entrenamiento.

\section{Empoderar a la gente}

- $\left.\quad{ }^{*}\right)$ Mi organización reconoce a la gente que toma la iniciativa.

- Mi organización da a la gente control sobre los recursos que necesitan para cumplir con su trabajo.

- Mi organización apoya a los empleados que toman riesgos calculados.

\section{Conectar a la organización}

- Mi organización anima a la gente a pensar desde una perspectiva global.

- $\quad\left(^{*}\right)$ Mi organización trabaja conjuntamente con la comunidad exterior para resolver necesidades mutuas.

- Mi organización alienta a la gente a que consiga respuestas a través de toda la organización para solucionar los problemas.

\section{Liderazgo estratégico para el aprendizaje}

- En mi organización, los líderes aconsejan y entrenan a los que dirigen.

- $\quad\left(^{*}\right)$ En mi organización, los líderes buscan continuamente oportunidades de aprender.

- En mi organización, los líderes se aseguran de que las acciones de la organización sean consistentes con sus valores.

En la práctica se ha utilizado el DLOQ para diagnosticar el aprendizaje estratégico en una asociación profesional (Milton, 2003). Sirvió para realizar el diagnóstico de la cultura de aprendizaje de una organización de servicio australiano (Dymock, 2003). McHargue (2003) lo utilizó para indagar la posibilidad de desarrollar una organización que aprende en entidades sin fines de lucro. En un sector público de Malasia la investigadora Sta. María
(2003) demostró que la cultura de aprendizaje (DLOQ) era la dimensión que más se relacionaba $(\mathrm{R} 2=.32)$ con la implementación eficaz del ISO 9001. En la ciudad de Bogotá, Hernández (2003) efectuó una investigación en ocho empresas y encontró que la cultura de aprendizaje influía en la transferencia del conocimiento tácito, y este a su vez tenía una influencia positiva en la mejora del rendimiento. Este autor efectuó primero la traduc- 
ción, la validación y la adaptación del DLOQ al español (Hernández \& Watkins, 2003).

Una de las pocas investigaciones que han indagado sobre la relación entre la cultura de aprendizaje y el rendimiento financiero de las empresas es la realizada por Ellinger et al. (2003). Ellos aplicaron el DLOQ a gerentes de logística de 208 empresas norteamericanas, y los puntajes obtenidos los relacionaron con cuatro medidas objetivas de rendimiento financiero de las empresas: valor de mercado añadido (MVA), retorno en equidad (ROE), retorno en activos (ROA), y el ' $\mathrm{q}$ ' de Tobin. El análisis de los datos mostró que más del $10 \%$ de la varianza en los cuatro indicadores del desempeño financiero podía ser explicado por las dimensiones del DLOQ, que si se tiene en cuenta "... la multitud de factores internos y externos que afectan el rendimiento financiero de una empresa (...), es muy significativo" (Ellinger et al., 2003, p. 167). Esto demuestra cómo una variable "blanda" (cultura de aprendizaje) influye en una variable más objetiva.

Como se puede constatar, el DLOQ ha demostrado ser útil en diversos países y culturas. Esto es un avance importante en el área de las 'organizaciones que aprenden' porque puede ayudar a superar una etapa de confusión que se ha ido incrementando progresivamente, al haber sido objeto de investigación de diversas disciplinas, como se evidencia en el Handbook of Organizational Learning and Knowledge (Dierkes, Berthoin-Antal, Chile, \& Nonaka, 2001; citado en Friedman, Lipshitz, \& Popper, 2005) que incluye capítulos separados para cada una de las disciplinas que lo analiza: psicología, sociología, ciencia administrativa, economía, antropología, ciencia política e historia. Con el DLOQ se lograría operar el constructo 'organización que aprende', y de esta manera avanzar más en su estudio, como sucedió con la operación de variables que en una época fueron muy controversiales, como es el caso de la variable inteligencia que se operó con el uso de los tests de inteligencia. Además, el DLOQ puede ser útil para diagnosticar la capacidad de cambio de una organización, al existir una estrecha relación entre cambio y aprendizaje, punto que analizaremos más adelante.

Recientemente, Small e Irvine (2006) desarrollaron una herramienta de diagnóstico de las organizaciones que aprenden, elaborado a partir de un método complejo de observación y registro de las conversaciones que efectúan los sujetos. Este método fue elaborado a partir del trabajo realizado por Pilkington (1999) en el área educativa (citado en Small \& Irving, 2006). El inconveniente de dicha herramienta es que se requiere un entrenamiento intensivo antes de su utilización. Los autores en mención han utilizado su método para indagar textos de especia- 
listas que tratan el tema, y han llegado a la conclusión de que hay tres condiciones básicas para la existencia de una 'organización que aprende': aprendizaje continuo, desarrollo de la gente y escuchar a los clientes.

\section{Desarrollo de una 'organización QUE APRENDE'}

Sobre la base de lo expresado hasta ahora, podemos señalar que el desarrollo de una 'organización que aprende' es un proceso de cambio sistemático e intencional para incrementar en la organización el 'aprendizaje organizacional' de alta calidad.

Existen diversas propuestas sobre cómo lograr convertirse en una 'organización que aprende'. La primera significativa la podemos identificar con la contribución de Peter Senge (1992, 1995) que originó un gran entusiasmo entre los practicantes, y sirvió de estímulo para que otros elaboraran propuestas prácticas para incrementar la capacidad de aprendizaje de la organización, como lo ejemplifica la obra de Guns (1996) para desarrollar una organización de rápido aprendizaje, los 10 pasos sugeridos por Kline y Saunders (1993), o la perspectiva de Garvin (1993). Muchas de estas propuestas eran sugerencias aisladas y de aplicación generalizada sin considerar las peculiaridades de las organizaciones.

Actualmente se tiene una mayor conciencia de la complejidad de los intentos para desarrollar una organización desde la perspectiva del aprendizaje, y se considera que es fundamental la participación de los trabajadores (Poell et al., 2004); los aprendizajes que se pueden lograr por imposición mayormente no perduran en el tiempo, constituyendo aprendizajes organizacionales de baja calidad (Ben-Horin, 2004).

Se recomienda que se inicie el proceso de cambio con un diagnóstico, principalmente de la cultura de aprendizaje imperante en la organización, y de manera participativa establecer objetivos de mejora, planes de acción correspondientes, y finalmente proceder con su implementación (Drejer, 2000). Para el diagnóstico es recomendable utilizar el DLOQ o el instrumento de Tannembaun (1997), por sus cualidades psicométricas. Estos permitirían identificar los niveles alcanzados en cada dimensión de los instrumentos, y planificar luego acciones concretas para superar los bajos niveles detectados.

Constituye un aporte interesante para el desarrollo del trabajo de Chen (2005), quien por un lado elabora un modelo sistémico de aprendizaje organizacional, conformado por nueve subsistemas: descubriendo, innovando, seleccionando, ejecutando, transfiriendo, reflexionando, adquiriendo conocimiento, contribuyendo con conocimiento, y construyendo una memoria organizacional. Por otro lado, identifica 35 herramientas y prácticas admi- 
nistrativas, como: rotación laboral, sistema de mentoría, encuestas de satisfacción laboral, planeamiento de escenarios, feedback de 360 grados, pensamiento opuesto, etcétera. Luego señala el posible impacto de estos en cada subsistema, de manera que facilite su selección según las necesidades de intervención de acuerdo con un diagnóstico previo. Proporciona también ejemplos de empresas que han aplicado las diversas herramientas.

Otro aporte importante es el de Yeung et al. (2000), quienes logran integrar teoría y propuestas prácticas para la acción, que incluyen una variedad de medidas administrativas para incrementar la capacidad de aprendizaje organizacional, que se basaría en la generación y difusión de ideas con "impacto", entendiéndose esto último como la capacidad para “... agregar valor a los diversos intereses de la empresa (inversionistas, clientes y empleados)" (p. 14). El modelo de estos autores incluye diversas secuencias que se inician con un diagnóstico de las capacidades e "incapacidades" actuales de la organización para el aprendizaje, hasta la toma de acciones concretas.

\section{LA 'ORGANIZACIÓN QUE APRENDE' Y SU RELACIÓN CON OTRAS METODOLOGÍAS DE CAMBIO}

Antes de proceder con el análisis de la relación entre el aprendizaje organizacional y otras metodologías de cambio, precisaremos qué entendemos por cambio y su relación con el aprendizaje.

Existe cambio cuando hay diferencias entre un antes y un después; de acuerdo con esto, podemos decir, por ejemplo, que una persona ha cambiado porque ahora piensa de modo distinto de lo que sostenía hace un año. Pero este tipo de cambio, en el campo únicamente cognitivo, no es el que interesa más, sino lo observado en la conducta. De este modo, habrá cambio si ahora se hacen las cosas de manera diferente de como se hacía en el pasado. Por otro lado, anteriormente se afirmó que identificamos el aprendizaje como el cambio de conducta relativamente permanente. Así, cambio y aprendizaje están muy vinculados, pero a la vez también presentan diferencias.

Cambio y aprendizaje difieren porque en todo aprendizaje siempre hay cambio, mientras que en un cambio puede haber o no aprendizaje. Por ejemplo, un futbolista en una oportunidad puede efectuar una jugada similar a la que hace el mejor jugador del mundo, pero después nunca más la puede repetir; en este caso hubo cambio pero no aprendizaje, porque faltó la estabilidad de la conducta a lo largo del tiempo.

Cambio y aprendizaje se influyen mutuamente. La necesidad de cambio puede ser un estímulo para propiciar diversos aprendizajes, que permitirán hacer las cosas de manera diferente. De igual manera, ciertos aprendizajes pue- 
den ser el origen de determinados cambios (Bhatt, 2000).

Después de haber hecho las precisiones necesarias sobre los conceptos de cambio y aprendizaje, estamos en condiciones de afirmar que en todas las metodologías de cambio organizacional estas serán exitosas en la medida en que fomenten aprendizajes organizacionales de alta calidad. La diferencia entre las metodologías consistirá en el tipo de estrategia que utilicen: énfasis en el aspecto tecnológico, factor humano, diseño estructural, etcétera. A pesar de estas diferencias, el enfoque de las organizaciones que aprenden pueden facilitar la implementación de las distintas metodologías de cambio. A continuación se analizará, desde la perspectiva de la 'organización que aprende' algunas metodologías de cambio.

\section{Estándar de calidad ISO 9001 y Administración de la Calidad Total (TQM)}

Actualmente, la mayoría de las organizaciones laborales otorga importancia a la calidad como una estrategia competitiva, siendo el ISO 9001 una de las certificaciones internacionales de mayor popularidad, que sirve como una garantía de que la organización diseña y ejecuta sus procesos en función de la satisfacción de necesidades de sus clientes. La Administración de la Calidad Total (Total Quality Management, TQM) es una filosofía y una práctica de la gestión de la organización, que tiene como principios rectores: la satisfacción del cliente como máxima prioridad, la mejora continua de los procesos y actividades, el empoderamiento y compromiso de todo el personal $\mathrm{y}$, finalmente, basarse en hechos (Sabolainen, 2000).

Las organizaciones que desean obtener la certificación según los estándares de calidad ISO 9001 tienen que realizar una serie de cambios en su forma de trabajo, y establecer registros de sus principales actividades, de manera que una auditora externa pueda realizar los seguimientos como base para la certificación, según los parámetros establecidos por la International Standards Organization (Hill, Hazlett, \& Meegan, 2001).

La adhesión de la organización al IS0 9001 puede ser una oportunidad para propiciar los aprendizajes, a partir de la indagación de cómo se están realizando las actividades y qué se requiere modificar para efectuar mejoras. Es una ocasión para que la organización realice una autoevaluación e identifique sus fortalezas y debilidades, además de permitir a las personas innovar. Marcus y Naveh (2005) indican que a través de los diálogos frecuentes y en la interacción entre lo explícito (nuevas reglas) y lo tácito (rutinas, modelos mentales basados en la práctica) se va creando un nuevo conocimiento, y hace posible adquirir competencias difíciles de imitar. Pero todo esto hay que considerarlo 
como posibilidad, debido a que se requieren condiciones básicas para que esto suceda. Se necesita un liderazgo visionario e inspirador, de manera que "... gane los corazones y la mente de los miembros de la organización" (BenHorin et al., 2004, p. 468) y se oriente a desarrollar una organización más eficiente, que no se limite solo a conseguir la certificación. Además, se requiere una cultura de aprendizaje, como lo demostró la investigación de Sta. María (2003), realizada en un sector público de Malasia para la aplicación del ISO 9001.

La búsqueda de la certificación IS0 9001 tiene un componente operacional, vinculado a la realización de las actividades laborales, y un componente de cultura organizacional conformado por valores y creencias que influyen en la motivación y el accionar de los sujetos (Savolainen, 2000). El enfoque de las organizaciones que aprenden puede ser útil al permitir centrarnos en la dimensión difícil de identificar pero que tiene un impacto crucial: la cultura de aprendizaje. Si la cultura no apoya el aprendizaje es probable que el sistema organizacional asimile el IS0 9001 de manera que se hagan cambios para no cambiar, es decir, adoptar formalismos y acciones para contrarrestar la carga burocrática que normalmente ocasiona el IS0 9001.

El IS0 9001 no es bueno o malo en sí, hay que considerarlo como una especie de "herramienta" (Ben-Horn et al., 2005), cuyo aporte dependerá de cómo se utilice, según las condiciones de la organización, teniéndose en cuenta que su implementación requiere tiempo y compromiso de todos los empleados, lo cual hace que sea un proceso de cambio complejo. Tener en cuenta esto nos ayudará a entender el resultado, mayormente negativo, que Marcus y Naveh (2005) encontraron al revisar las investigaciones que indagaron sobre la utilidad del ISO 9000 para el rendimiento organizacional, a pesar de que en la práctica se ha venido aplicando en más de 100 países.

De lograrse buenos resultados con la aplicación del ISO 9001 se tendría una base sólida para la aplicación de la TQM en la organización, que requiere las mismas condiciones señaladas para el ISO 9001, pero con la diferencia de que el liderazgo destaca por su capacidad e interés por aprender y servir de modelo a los demás (Hill et al., 2001). La dimensión liderazgo debe ser considerada de manera amplia, incluyendo en ella a muchas personas, especialmente aquellas que tengan una personalidad orientada hacia el aprendizaje (Button \& Mathieu, 1996), al ser más receptivas con este cuando haya un medio ambiente que lo estimule (Farr, Hofmann, \& Ringenbach, 1993). Al hacerse una selección para determinadas tareas grupales, las personas que posean dicha personalidad deberán ser los primeras en ser consideradas. 


\section{Indagación Apreciativa}

El psicólogo David Cooperrider desarrolló la metodología de cambio denominada Indagación Apreciativa (IA), que se caracteriza por estar centrada en el aprendizaje de las experiencias positivas. Inicialmente, Cooperrider, cuando era estudiante de doctoral, en 1980, observó en una clínica que el aprendizaje de lo positivo permitía obtener mejores resultados en el cambio organizacional (Watkins \& Mohr, 2001). Este descubrimiento lo motivó para indagar más y desarrollar una sustentación teórica un tanto compleja, al recurrir a otras disciplinas, como la física cuántica, la teoría del caos, la sociología del conocimiento, e investigaciones sobre el poder de la imagen (Cooperrider et al., 2003).

Desde un punto de vista operativo la IA comprende básicamente lo siguiente: se reúnen todos los trabajadores y los ejecutivos de la empresa y conforman subgrupos en los que recuerdan los acontecimientos más positivos de su trabajo (descubrimiento), luego imaginan cómo podría ser su trabajo si dichos acontecimientos se presentaran de manera habitual (sueño), en tercer lugar se ponen de acuerdo sobre los cambios para incrementar la frecuencia de lo positivo (diseño), y finalmente se determina cómo se ejecutarán en la práctica (entrega).

Al proceder de la manera descrita se acelera el cambio al no estar separados el diagnóstico y la acción. El énfasis en lo positivo incrementa las emociones favorables y la motivación de los participantes. Además, al indagar sobre lo positivo aumenta su presencia en el futuro, mientras que al considerar lo negativo este se incrementa más. (Bushe \& Coetzer, 1995).

Desde la perspectiva del aprendizaje organizacional la IA es un enfoque que otorga una relevancia sustancial al aprendizaje, de manera que puede ser clasificada como una modalidad específica para conseguir el desarrollo de una organización que aprende.

En nuestro medio, la IA ha tenido escasa presencia, a pesar de que no es una metodología reciente, debido posiblemente a que sus principios básicos van en contra de la forma habitual de hacer los diagnósticos organizacionales, centrados desde un comienzo en los problemas.

\section{Medición y mejora de la productivi- dad Laboral: Metodología ProMES}

Robert Pritchard (1990) ha desarrollado una metodología de mejora laboral denominada Productivity Measurement and Enhancement System (ProMES). Landy (2005) la describe como una metodología exitosa para incrementar la motivación laboral del personal.

La ProMES se caracteriza por otorgar participación a los trabajadores, quienes junto con sus jefes y facilitadores elaboran un sistema de medida 
sobre las contribuciones de su área a la empresa; luego se aplica dicha medida, y los resultados son divulgados y sirven de base para diseñar acciones de mejoras. Después de obtenerse la estabilidad del sistema en la organización se implementa un programa de recompensas (Solf, 1997).

En la actualidad dicha metodología es uno de los principales aportes de la psicología organizacional, y la “... evidencia acumulativa muestra ganancias significativas en la productividad después de la introducción de la ProMES" (Landy \& Conte, 2005, p. 375). Posee bases teóricas muy sólidas, sustentadas en la teoría de las metas (Locke \& Latham, 1990), el efecto de la participación, la influencia de la retroinformación y de los reforzadores positivos (Pritchard, 1990; Poell, 2004). Además, se puede integrar con los aportes del Cuadro de Mando o Balanced Scorecard (Kaplan \& Norton, 1997), que considera de manera específica una dimensión de indicadores relacionados con el aprendizaje y el crecimiento.

La presencia del aprendizaje en la metodología ProMES la podemos ubicar en la influencia motivacional de la cultura organizacional en el desempeño de los trabajadores, quienes son los informantes claves en la aplicación del sistema de medida, de manera que si no están convencidos del método pueden otorgar información no válida. De igual manera, después de acordar los cambios por ejecutar, como resultado del diagnóstico, los trabajadores son los principales responsables para lograr las metas sobre la base de nuevos aprendizajes. De acuerdo con esto, podemos deducir la importancia que tiene la cultura, y en especial lo vinculado con el aprendizaje. Para el éxito del cambio también se requieren otras condiciones, como la continuidad de los supervisores en la organización para no introducir interrupciones innecesarias, así como un nivel adecuado de confianza entre jefe y subordinado (Pritchard, 1995).

\section{REFERENCIAS}

Alcover, C. M. \& Gil. F. (2002). Crear conocimiento colectivamente: Aprendizaje organizacional y grupal. Revista de Psicología del Trabajo $y$ de las Organizaciones, 18 (2-3), 259-301.

Argyris, C. (2001). Sobre el aprendizaje organizacional (2. ${ }^{\mathrm{a}}$ edición). México: Oxford University Press.

Argyris, C. \& Schön, D. A. (1978). Organizational learning: A theory of action perspective. Reading, Ma.: Addison-Wesley.

Ben-Horin, Y.; Lipshitz, R. \& Popper, M. (2004). Discerning the Quality of Organizational Learning. Management Learning, 35(4), 451-471. 
Bhatt, G., (2000). Information dynamics, learning and knowledge creation in organizations. The Learning Organization, 7, 89-98.

Braham, B. (1995). Creating a Learning Organization. California: Crips.

Brooking, A. (1997). El capital intelectual. El principal activo de las empresas del tercer milenio. Barcelona: Paidós.

Bushe, G. R. \& Coetzer, G. (1995). Appreciative inquiry as a team development: A controlled experiment. Journal of Applied Behavioral Science, 31(1), 19-31.

Button, S. \& Mathieu, J. (1996). Goal Orientation in organizational research: A conceptual and empirical foundation. Organizational Behavior and Human Decision Processes, 67(1), 26-48.

Chen, G. (2005). Management practices and tools for enhancing organizational learning capability. S.A.M. Advanced Management Journal, 70(1), 4-35.

Choo, C. W. (1999). La organización inteligente. México: Oxford University Press.

Cooperrider, D. L.; Whitney, D., \& Stavros, J. M. (2003). Appreciative Inquiry Handbook. San Francisco: Berrett-Koehler Publishers, Inc.
Dixon, N. (1999). The Organizational Learning Cycle. How we can learn collectively. Vermont: Gower.

Drejer, A. (2000). Organizational learning and competence development. The Learning Organization, 7, 206220.

Dymock, D. (2003). Developing culture of learning in changing industrial climate: An Australian case study. Advances in Developing Human Resources, 5(2), 185-195.

Ellinger, A. D.; Ellinger, A. E.; Yang, B., \& Howton, S. W. (2003). Making the business case for the learning organizational concept. Advances in Developing Human Resources, 5(2), 163-172.

Farr, J. L., Hofmann; D. A., \& Ringenbach, K. L. (1993). Goal Orientation and action control theory: Implications for Industrial and Organizational Psychology. En: C. L. Cooper \& I. T. Roberston (Eds.). International review of industrial and organizational psychology, Chichester: John Wiley.

Friedman, V. J.; Lipshitz, R., \& Popper, M. (2005). The mystification of organizational learning. Journal of Management Inquiry, 14(1), 19-30.

Fruin, W. M. (2000). Las fábricas del conocimiento. La administración del capital intelectual en Toshiba. México: Oxford University Press. 
Garvin, D. (1993). Building a Learning Organization. Harvard Business, (July-Aug.).

Gore, E., \& Dunlap, D. (2006). Aprendizaje y organización. Una lectura de teoría de la organización. Buenos Aires: Granica.

Guns, B. (1996). Aprendizaje Organizacional. Cómo ganar y mantener la competitividad. México: Prentice-Hall.

Hernández, M. (2003). Assessing tacit knowledge transfer and dimensions of a learning environment in colombian businesses. Advances in Developing Human Resources, 5(2), 215-221.

Hernández, M. \& Watkins, K. E. (2003). Translation, validation and adaptation of the Spanish version of the modified Dimensions of the Learning Organization Questionnaire. Human Resource Development International, 6(2), 187-196.

Hill, F. M.; Hazlett, S.A. \& Meegan S. (2001). A study of the transition from ISO 9000 in the context of organizational learning. The International Journal of Quality \& Reliability Management, 12(2), 142-152.

Kaplan, R. S., \& Norton, D. P. (1997). El cuadro de mando integral. The balanced scorecard. México: Gestión, 2000.
Kline, P., \& Saunders, B. (1993). Ten steps to a Learning Organization, Virginia: Great Ocean.

Kolb, D. A., Rubin, I. M. \& McIntyre, J. M. (1974). Psicología de las organizaciones: Experiencias. México: Prentice-Hall.

Landy, F. J., \& Conte, J. M. (2005). Psicología industrial. Introducción a la psicología industrial y organizacional. México: McGraw-Hill.

Locke, E. A., \& Latham, G. P. (1990). A theory of goal setting \& task performance. Nueva Jersey: PrenticeHall.

Koulopoulos, T., \& Frappaolo. (2000). Lo fundamental y lo más efectivo acerca de la Gerencia del Conocimiento. Bogotá: McGraw-Hill.

Krogh, G. von, Ichijo, K., \& Nonaka, I. (2001). Facilitar la creación de conocimiento. Cómo desentrañar el misterio del conocimiento tácito y liberar el poder de la innovación. México: Oxford University Press.

March, J. G., \& Simon, H. (1958). Organizations. Nueva York: McGraw-Hill.

Marcus, A., \& Naveh, E. (2005). How a new rule is adjust to context: knowledge creation following the implementation of the ISO 9000 quality standard. International Journal of Organizational Analysis, 13 (2), 106-126. 
Marsick, V. J. \& Watkins, K. E. (2003). Demostrating the value of an organization's learning culture: The Dimensions of the Learning Organization Questionnaire. Advances in Developing Human Resources, 5(2), 132-151.

Mayo, A., \& Lank, E. (1994). Las organizaciones que aprenden. Barcelona: Gestión, 2000.

McHargue, S. K. (2003). Learning for performance in nonprofit organizations. Advances in Developing Human Resources, 5(2), 196-204.

Milton, J. (2003). Professional associations as learning systems: Learning + Strategy + Action $=$ Strategic Learning. Advances in Developing Human Resources, 5(2), 173-181.

Moilanen, R. (2005). Diagnosing and measuring learning organizations. The Learning Organization, 12(1), 71-89.

Nonaka, I. \& Takeuchi, H. (1999). La organización creadora de conocimiento. México: Oxford University Press.

O'Brien, M. (1994). Learning Organization Practices Profile. California: Pfeiffer \& Company.

Pedler, M.; Burgoyne, J. \& Boydell, T. (1997). The Learning Company. Londres: McGraw-Hill.

Poell, R. F.; Van Dam, K. \& Van den Berg, P. T. (2004). Organising
Learning in Work Contexts. Applied Psychology an International Review, 53(4), 529-540.

Pritchard, R. D. (Ed.). (1995). Productivity measurement and improvement. Organizational case studies. Nueva York: Praeger.

Pritchard, R. D. (1990). Measuring and improving organizational productivity. A Practical guide. Nueva York: Praeger.

Sabolainen, T. (2000). How organizations promote and avoid learning: Development of positive and negative learning cycles. Journal of Workplace Learning, 12(5), 195-207.

Senge, P. (1992). La quinta disciplina. Barcelona: Granica.

Senge, P.; Ross, R.; Smith, B. \& Kleiner, A. (1995). La quinta disciplina en la práctica. Barcelona: Granica.

Small, A. \& Irvine, P. (2006). Towards a framework for organizational learning. The Learning Organization, 13(2-3), 276-298.

Solf, A. G. (2003). Desarrollo organizacional desde la perspectiva del aprendizaje. Avances en Psicología, octubre. Lima: Unifé.

Solf, A. G. (1997). Medición y mejora de la productividad laboral: Metodología ProMES. Avances en Psicología Organizacional, Octubre. Lima: Unifé. 
Sonnetang, S.; Niessen, C., \& Ohly, S. (2004). Learning at work: Training and development. En: C.L. Cooper \& I. T. Roberstson (Eds.). International Review of Industrial and Organizational Psychology. Vol. 19, 249-289.

Sta. María, R. F. (2003). Innovation and organizational learning culture in the malaysian public sector. Advances in Developing Human Resources, 5(2), 205-214.

Swieringa, J. \& Wierdsma, A. (1995). La organización que aprende. México: Addison-Wesley.

Tannenbaum, S. (1997). Enhancing continuous learning diagnostic findings from multiple companies. Human Resource Management, 36, 437-452.

Thomas, K., \& Allen, S. (2006). The learning organization: A metaanalysis of themes in literature. The Learning Organization 13, (2-3), 123-139.
Watkins, J. M. \& Mohr, B. J. (2001). Appreciative Inquiry. Change at the speed of imagination. San Francisco: Jossey-Bass/Peiffer.

Watkins, K. E. \& Marsick, V. J. (1998). Dimensions of the Learning Organization Questionnaire. Warwick, RI: Partners for the Learning Organization.

Yang, B. (2003). Identifying valid and reliable measures for dimensions of a learning culture. Advances in Developing Human Resources, 5 (2), 152-162.

Yeo, R. K. (2005). Revisiting the roots of learning organization. A synthesis of the learning organization literature. The Learning Organization, 12(4), 368-375.

Yeung, A.; Ulrich, D.; Nason, S. \& Glinov, M. (2000). Las capacidades de aprendizaje en la organización. México: Oxford University Press. 
Solf Zárate 